\title{
Análisis
}

\section{El campus de la Universidad Carlos III en Second Life}

\author{
Por Francisco López-Hernández
}

\begin{abstract}
Resumen: Second Life es un entorno virtual en tres dimensiones que muchos consideran simplemente un juego pero que tiene enormes posibilidades para la enseñanza a distancia y la comunicación. Hay bibliotecas universitarias que están intentando aprovecharlo en este sentido; la de la Universidad Carlos III es una de ellas. En este artículo se presenta su proyecto, en el que pretende anticiparse a lo que muy probablemente sea la forma de presentación futura de la información en internet: la Web 3D.

Palabras clave: Metaversos, Second Life, Bibliotecas universitarias, Enseñanza a distancia, Web 2.0, Web 3D.

Title: The Universidad Carlos III campus in Second Life

Abstract: Second Life is a three-dimensional virtual environment that many consider merely a game but it has enormous possibilities for e-

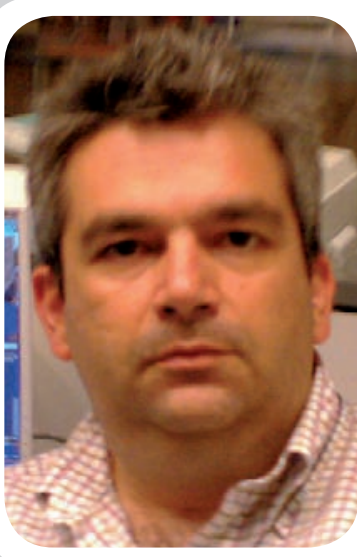

Francisco López-Hernández es licenciado en farmacia y trabaja en bibliotecas desde 1993, siempre en la Universidad Carlos III. Es coordinador del Servicio de Atención a Usuarios de la biblioteca del Campus de Colmenarejo y coordina el grupo de trabajo del proyecto de campus virtual de la Universidad Carlos III en Second Life.

learning and communication. There are academic libraries that are trying to take advantage of it; the Universidad Carlos III Library is one of them. This paper presents its project, with which it expects to anticipate that which very probably will be the future way of displaying information on the internet: $3 D$ web.
\end{abstract}

Keywords: Metaverses, Second Life, Universitary libraries, e-Learning, Web 2.0, 3D Web.

López-Hernández, Francisco. "El campus de la Universidad Carlos III en Second Life”. En: El profesional de la información, 2008, noviembre-diciembre, v. 17, n. 6, pp. 657-661.

DOI: $10.3145 /$ epi.2008.oct.08

\section{¿Qué es Second Life?}

SEGÚN LA PROPIA EMPRESA QUE LO CREÓ y lo mantiene (Linden Lab, fundada en 1999 por Philip Rosedale), Second Life es "un mundo virtual en tres dimensiones construido por sus residentes"1.

Se puso en marcha en 2003 y durante un tiempo vivió una existencia un tanto lánguida, con un escaso incremento de usuarios, hasta que en los años 2006-2007 se produjo una explosión que hizo que el número de cuentas registradas pasase de 95.000 (enero de 2006) a 7,4 millones (junio de 2007) ${ }^{2}$. Ese mismo año 2007 empezó en EUA una campaña de descrédito, alentada sobre todo por tres artículos publicados en el periódico Los Angeles times y en las revistas Wired y
Forbes en los que se insinuaba que Second Life era un fracaso para las empresas que tenían presencia allí porque entre otras cosas, estaba medio vacío ${ }^{3}$. En España la prensa, que poco antes había hablado maravillas de este mundo virtual, pasó al poco tiempo a denostarlo e incluso a insinuar que no era más que un lugar para ganar dinero sin trabajar o cometer perversiones sexuales ${ }^{4}$.

En el ámbito español aún hay personas que piensan así, la mayoría porque no conocen realmente qué es Second Life y las posibilidades que ofrece en el campo de la comunica-

\section{Metaverso}

Término que viene de la novela Snow Crash publicada en 1992 por Neal Stephenson. Se usa para describir la visión del trabajo en espacios 3D de realidad virtual totalmente inmersivos. Los metaversos son entornos donde los humanos interactúan social y económicamente como iconos a través de un soporte lógico en un ciberespacio que es una metáfora del mundo real, pero sin limitaciones físicas.

\section{Fuente: Wikipedia}

http://es.wikipedia.org/wiki/Metaverso 


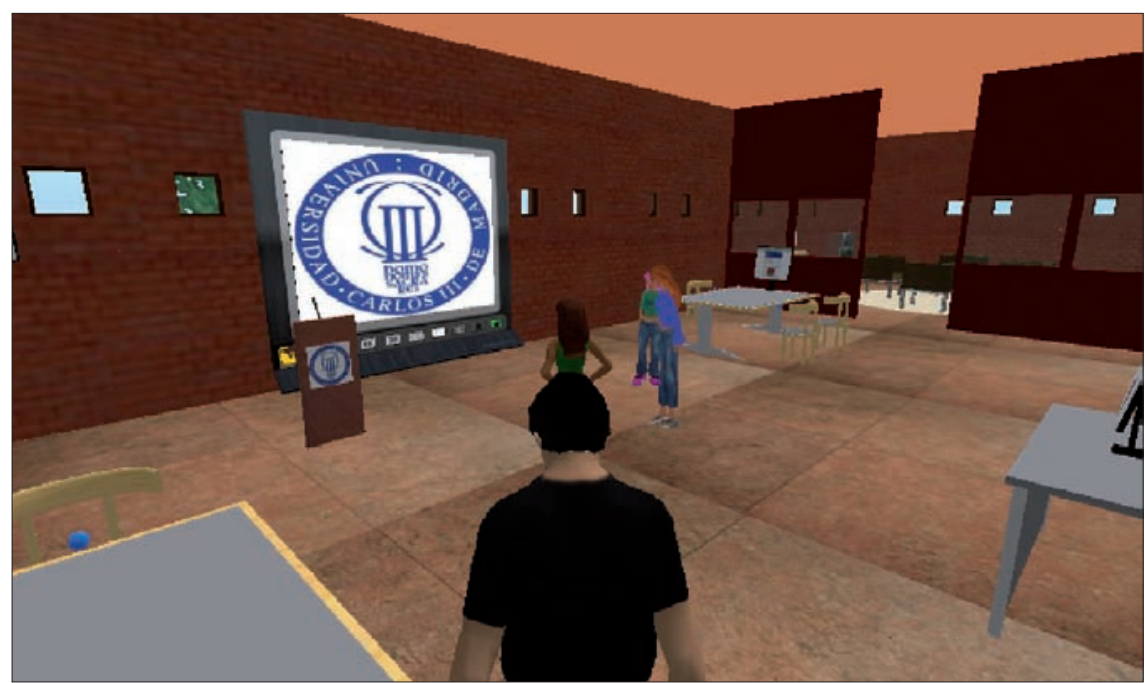

Aula de trabajo en grupo del edificio principal

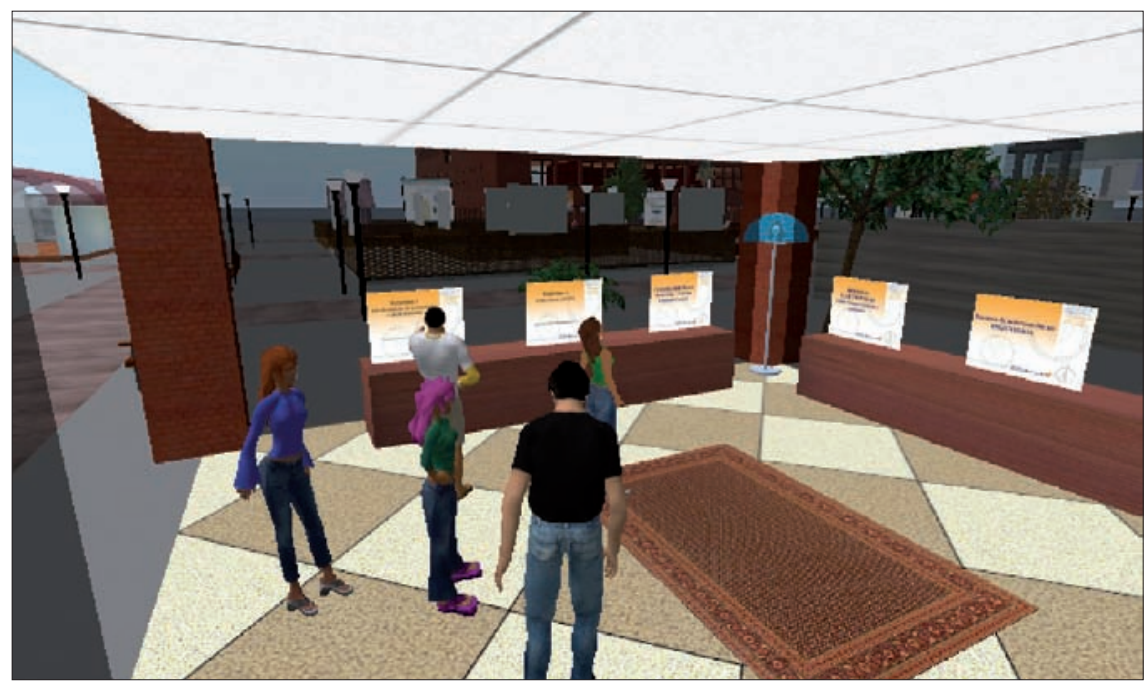

Edificio de formación especializada en Ciencia y Tecnología (zona de enlaces a tutorías de autoaprendizaje)

ción y de la educación a distancia. Se han quedado con la idea de que "casi todo el mundo se da de alta y de inmediato se aburre y se marcha", cuando seguramente desconocen que el movimiento en contra de este metaverso ya ha dado la vuelta en EUA y que el número de iniciativas interesantes va creciendo, sin prisa pero sin pausa, dentro de él $1^{5}$.

El mundo virtual de Second Life está organizado en forma de islas o sims (de simuladores) que pueden estar aisladas, unidas a un continente o formando un archipiélago. Cualquiera puede poseer una isla completa o bien una parcela, desde $1 / 128$ de su superficie (que a escala son $65.535 \mathrm{~m}^{2}$ ).

\section{Posibilidades que ofrece Second Life en la enseñanza en línea: Sloodle}

Es cierto que muchos usuarios utilizan Second Life como un mero videojuego. Cuando se pasea por el metaverso se pueden escuchar muchas conversaciones típicas de un chat de adolescentes. Pero también hay mucha gente que desarrolla incluso una carrera profesional allí, y no sólo en el campo que nos interesa a nosotros: desde ocupaciones más artesanales como la creación de muebles, ropa o complementos para los avatares hasta parques empresariales pensados para aquellos que tienen iniciativa ${ }^{6}$.
"Hay mucha gente que realiza incluso una carrera profesional en Second Life, y no sólo en el campo que nos ocupa a nosotros"

Entre las opciones que a los bibliotecarios y profesionales de la información pueden interesar cabe citar:

- Enlaces a páginas web ${ }^{7}$.

- Vídeo (en diferido y también en directo, vía streaming).

- Presentaciones (síncronas y asíncronas).

- Imágenes estáticas (exposiciones).

- Chat escrito y comunicación por voz (referencia en línea).

- Referencia asíncrona (correo electrónico, mensajes, tablones de anuncios).

- Encuestas.

- Merchandising: venta o regalo de camisetas para avatares, y de otros objetos.

- Clases y reuniones virtuales.

- Y todo ello en un entorno tridimensional agradable, sencillo y fácil de utilizar, que además es muy familiar para los jóvenes.

Uno de los frutos más interesantes que ha producido esta visión de Second Life como importante vehículo para la enseñanza a distancia es Sloodle. Se trata de una aplicación web híbrida (un mashup o integración de varios servicios) de código abierto, que pretende utilizar los entornos virtuales en tres dimensiones para el aprendizaje. Para ello se busca una convergencia entre las posibilidades de Second Life y las de la conocida plataforma de enseñanza a distancia Moodle M $^{\text {. }}$ 


\section{"Uno de los frutos más interesantes que ha producido Second Life como importante vehículo para la enseñanza a distancia es Sloodle"}

\section{La biblioteca de la Universidad Carlos III en Second Life}

Aprovechar las posibilidades antes enumeradas o al menos algunas de ellas, es precisamente lo que pretende el proyecto que desde enero de 2008 está llevando a cabo la biblioteca de la Universidad Carlos III en Second Life y que comenzó con la adquisición de una isla9. Se planteó como un objetivo en el marco del Plan Estratégico de la biblioteca para 2008-11 y se creó un grupo de trabajo en el que participaron tanto bibliotecarios como informáticos.

El proyecto ha de ser dinámico y experimentar cambios constantes, por lo cual no sería correcto decir que a estas alturas está terminado. Sí se podría afirmar que todos los servicios que pretendía dar la biblioteca ya se pueden ofrecer a junio de 2008. Ahora falta que otras unidades de la universidad tengan presencia allí (el Parque Científico Leganés Tecnológico, por ejemplo, ya tiene su edificio preparado).

\section{"El proyecto ha de ser dinámico y ha de evolucionar constantemente, por lo cual nunca está terminado"}

Quienes conozcan bien Second Life y visiten este campus tal vez

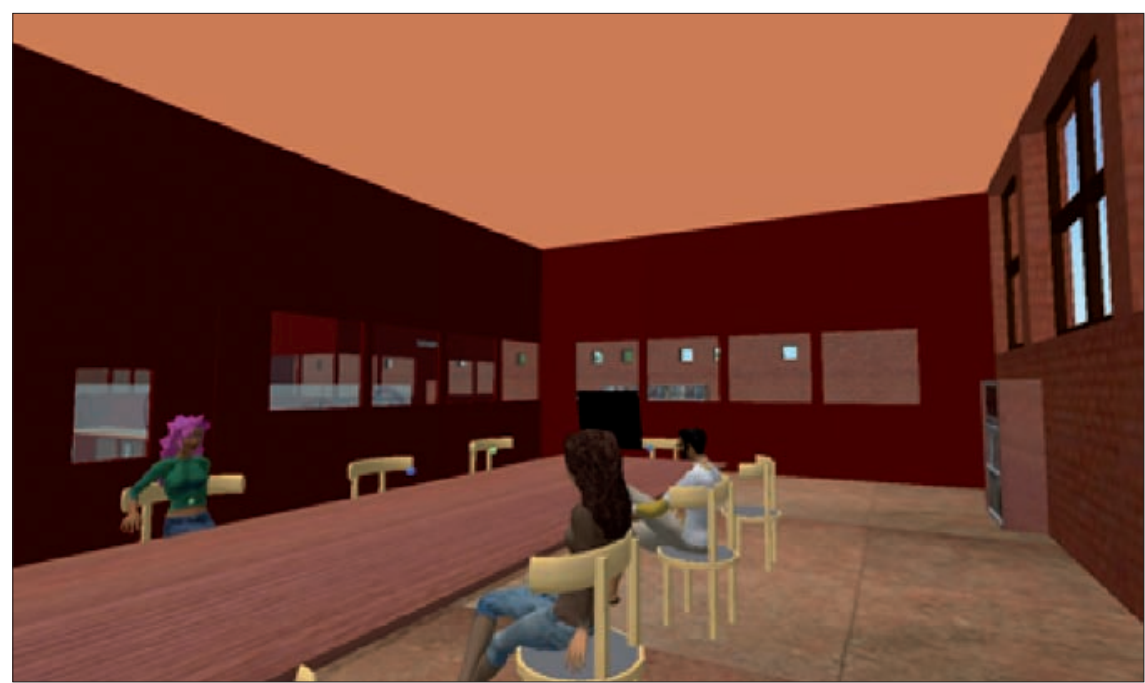

Reunión virtual en la sala de juntas del edificio principal

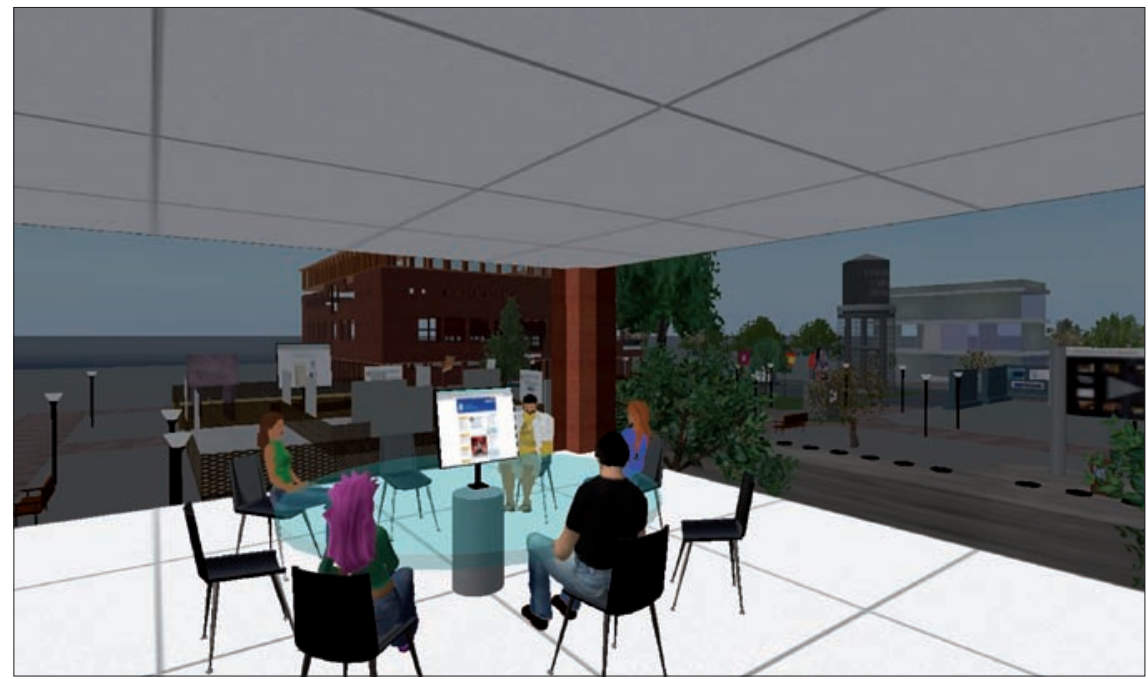

Ejemplo de sesión virtual de información en el edificio de Ciencia y Tecnología

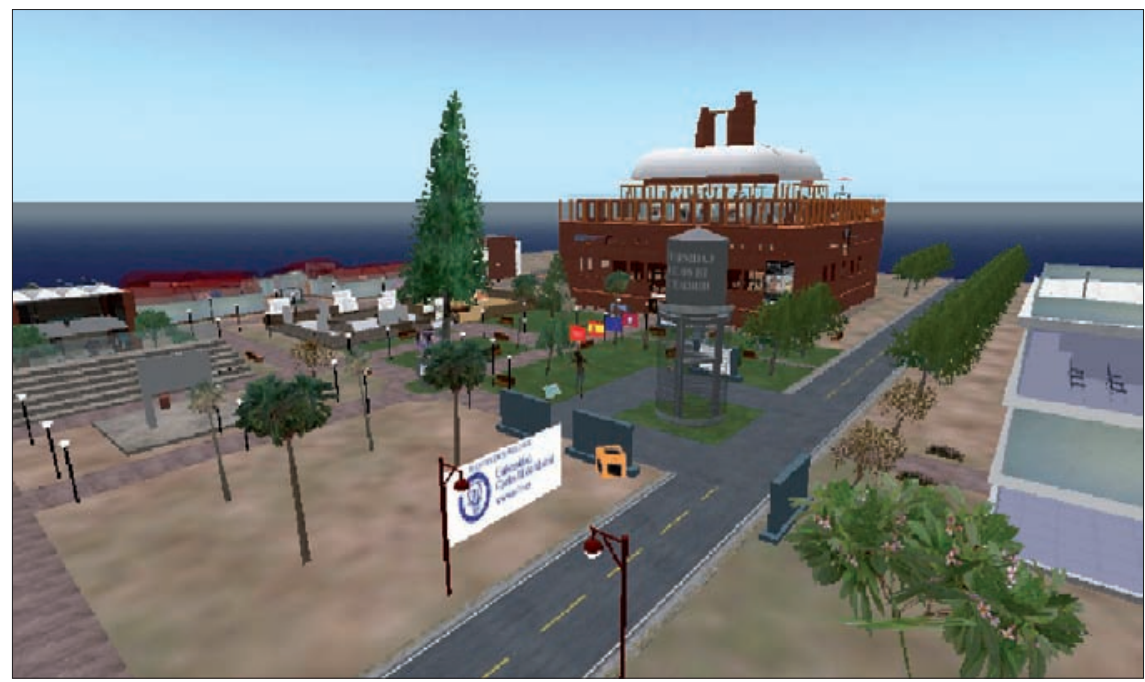

Vista general del campus de la UC3M en Second Life

puedan pensar que su apariencia es un tanto espartana si se compara con algunas otras iniciativas. En realidad no se ha buscado la espec- tacularidad visual, sino que los contenidos sean lo más ricos posible para sus usuarios potenciales. Esto es así porque, sin negar las posibi- 


\section{"Nos ha interesado implementar la formación y la referencia en línea para nuestros usuarios allá donde se encuentren"}

lidades mercadotécnicas que pueda tener este medio, son otros aspectos los que más nos ha interesado desarrollar, especialmente su utilización en la formación y la referencia en línea para nuestros usuarios allá donde se encuentren.

El centro de la isla es el edificio principal, que intenta reproducir, al menos en su apariencia externa, la biblioteca de la Escuela Politécnica Superior del campus de Leganés (edificio Rey Pastor). Está dispuesto en cuatro plantas. En la baja, además de un mostrador central de información, una zona para la prensa electrónica y otra reservada para exposiciones que organice la biblioteca, hay mesas para trabajar en grupo con pantallas de ordenador que enlazan con servicios tales como las bibliotecas depositarias (ONU, OIT), el Centro de Documentación Europea, el Taller del Aula, el Centro Patlib, y otro que recupera la bibliografía recomendada por titulaciones tal y como aparece en el catálogo de nuestra biblioteca. También hay un lugar reservado para la referencia en línea, donde encontramos monitores de presencia que indican si el avatar que nos atenderá está o no (en este último caso se le puede enviar un mensaje que le llegará por correo electrónico a la persona que está detrás de ese avatar).

La primera planta está destinada a sala de juntas para reuniones virtuales, a zona de trabajo con varias mesas que pueden utilizar los avatares de los estudiantes para trabajar en grupo y a dos aulas que se pueden emplear tanto para conferencias como para dar clase.
La segunda planta es semejante, con varios espacios de trabajo en grupo y un aula inspirada en las nuevas tendencias de clase dinámica que nos llegan de las universidades anglosajonas, con mesas para estudiar en común, ordenadores que se consultan de pie, grandes pantallas en las paredes y una tribuna para los casos en que alguien tiene que dirigirse a los demás.

La tercera planta es una galería de imágenes de las bibliotecas de nuestros tres campus "reales" y de todo el personal que trabaja allí.

La cuarta es una terraza que constituye la zona de recreo: una máquina de café, otra de refrescos (virtuales, por supuesto), mesas y sillas con sus correspondientes sombrillas, música y la posibilidad de que nuestros avatares se marquen un baile.

Fuera de este edificio hay diversos espacios. En primer lugar se ha intentado reflejar allí todo lo que vamos haciendo para tender a lo que conocemos como biblioteca 2.0; en concreto, los tres blogs que aquí se mantienen (365 días de libros, Biblioteca y + música y Videodrome) tienen allí su espacio, representado uno por un enorme carro de libros, otro presidido por la foto de una banda de jazz que invita al baile (cosa que, de hecho se puede hacer en su recinto) y el otro por una pequeña sala de cine.

También hay una zona pensada para realizar cursos básicos de acogida (denominados Curba en nuestro centro) en el Laberinto Curba, donde un avatar ha de recorrer el camino viendo los grandes carteles que le salen al paso (algunos de ellos con enlaces a páginas web) y donde se explica lo básico sobre cómo usar nuestra biblioteca de la vida real.

Más allá hay cuatro hangares informativos dedicados a las colecciones especiales (de idiomas, de Madrid, de viajes y fondo antiguo), antes de llegar al espacio de la formación especializada y autoaprendizaje.

Se han dispuesto tres edificios, cada uno dedicado a uno de los tres grandes grupos de materias que se estudian en la Universidad Carlos III: ciencia y tecnología, ciencias sociales y jurídicas, y humanidades, comunicación y documentación. Son tres edificios de diseño diferente pero con contenidos similares: enlaces a presentaciones power point pensadas como elementos de autoaprendizaje y que se han col-

\section{"Aunque no sea Second Life, parece claro que la interfaz futura de la Web será muy parecida: un entorno en tres dimensiones"}

gado en SlideShare ${ }^{10}$, enlaces a las recopilaciones de fuentes de información que ha hecho la biblioteca y también a las correspondientes guías de recursos electrónicos. Desde estos edificios, los avatares pueden teletransportarse al Laberinto Curba (si lo que realmente buscaban era un curso básico), recurrir al "pregunte al bibliotecario" mediante nuestro catálogo o solicitar un

\section{Avatar}

Palabra tomada del hinduismo, donde un avatar es la encarnación terrestre de un dios, en particular Vishnú.

Se dice por ejemplo que el dios Krishna es el octavo avatar de Vishnú. 
curso de formación del tipo que sea. En los tres edificios hay asimismo espacios para dar formación dentro del metaverso.

No nos alejaremos mucho para llegar al auditorio, con capacidad para 90 avatares (los asientos tienen un script que permite al que está sentado levantar la mano) y donde se puede ver el vídeo de promoción de nuestra biblioteca. Poco más allá, una gran sala de exposiciones y también un San$d b o x$, que es un espacio pensado para que los avatares puedan hacer pruebas de construcción y manejo de objetos.

Al otro lado de la carretera que divide en dos la isla se establecerán los demás servicios de la universidad. Allí está ya el mencionado edificio del Parque Científico y también uno dispuesto para los profesores de las titulaciones semipresenciales, que acaban de arrancar en nuestra universidad y que pueden ser unas de las principales beneficiarias de esta herramienta.

Se puede visitar esta isla mediante el siguiente enlace ${ }^{11}$ :

http://slurl.com/secondlife/Univers idad\%20Carlos\%20III/138/102/23

\section{Conclusión}

Aunque no sea exactamente Second Life, parece claro que la interfaz futura de la Web será muy parecida: un entorno en tres dimensiones mucho más apto para el entendimiento de los seres humanos que la actual presentación bidimensional. Bueno es por tanto ir acostumbrándonos e incluso trabajar en ello con anticipación ante lo que pueda llegar.

Reproduzco para terminar unas palabras del creador de Second Life Philip Rosedale, que van en este sentido: "La gran transición ha sido el camino desde la Web hasta la Web 3D (...). Mi perspectiva es que Second Life como tecnología produce dos cambios: en principio había textos conectados entre sí por hiperenlaces. La internet 3D como Second Life permite presentar la información de una manera perfecta para los seres humanos. La mayoría de las asociaciones se realizan utilizando símbolos universales. Una silla, por ejemplo, es un símbolo universal. Si puedes organizar la información con estos símbolos entonces estás llevando a cabo una verdadera transformación. Somos animales sociales. Así, Second Life da la posibilidad de compartir información con otros (consumir, crear, vender, etc.). Como mentes vivas, queremos contacto vivo con otras personas. Esta tecnología permite informarse, crear, consumir con otras personas en tiempo real. Eso es lo que estamos construyendo $\mathrm{y}$ es el cambio que trae la internet 3D"'12.

\section{Notas}

1. What is Second Life? Consultado en: 06-0608.

http://secondlife.com/whatis/

2. Los datos están tomados del mensaje que se encuentra en la web y que se pueden descargar en formato Excel.

http://blog.secondlife.com/2007/06/12/may2007-key-metrics-published/

3. Véase: Jau, Wagner James. Second Life backlash reversed? Former detractor now touting SL for enterprise using. En: New world notes. Consultado en: 06-06-08. En este mensaje el autor se refiere a los tres artículos mencionados y a cómo ahora quienes los escribieron piensan exactamente lo contrario.

http://nwn.blogs.com/nwn/2008/05/backlashrevers.html

4. Esto es lo que se lee por ejemplo en el artículo de Ramón Muñoz en El país de 15 de agosto de 2007, "Second life está desierto", en el que se dice textualmente, escudándose en lo indicado en la revista Wired: "la mayor parte de sus visitantes sólo buscan conseguir dinero gratis o practicar perversiones sexuales".

http://www.elpais.com/articulo/revista/agosto/ Second/Life/desierto/elpeputec/20070815elpepirdv $5 /$ Tes

5. Sin citar las innumerables iniciativas en EUA o el Reino Unido, en España universidades como la Autónoma de Madrid, Pública de Navarra, Politécnica de Madrid, La Coruña o la Miguel Hernández de Elche (entre otras) tienen o han tenido su espacio más o menos grande, más o me- nos público, más o menos ambicioso en Second Life. La Casa Encendida, el Instituto Cervantes, el Instituto de Comercio Exterior (ICEX) y, entre las empresas privadas, Seat o CCC (dedicada precisamente a la enseñanza a distancia) también han visto útil estar presentes en este metaverso.

6. Tal es el caso de Novatierra, impulsada por el incansable Luis Sotillos, "Luis Aldrich" en Second Life.

http://novatierra.com/

7. Hasta el momento (junio de 2008) los enlaces a páginas web se hacen mediante un script escrito en el lenguaje de programación $L S L$ (Linden Scripting Language, semejante a $C$ o Javascript) asociado a un objeto que al ser tocado por un avatar hace que aparezca un navegador interno donde se presenta la página (también se puede hacer que salga en uno externo). Está en marcha un proyecto llamado "Web on Prim" con el que será posible crear objetos en los que se pueda navegar directamente.

8. Una explicación más profunda sobre las posibilidades de Sloodle se escapa del alcance de este artículo. Para quienes sientan curiosidad, recomiendo la visita de su web.

http://www.sloodle.org

$\mathrm{Si}$ se quiere tener una imagen clara de lo que pretende ser, se puede consultar el gráfico que aparece en:

http://slisweb.sjsu.edu/sl/index.php/Sloodle Portada

9. En junio de 2008 el coste de una isla en Second Life para una entidad educativa o sin ánimo de lucro es de 700 US\$ en concepto de compra y de 147,50 US\$ al mes por mantenimiento. Los precios se pueden consultar en:

http://secondlife.com/land/privatepricing.php

10. Es un espacio web que sirve para compartir presentaciones power point a las que se pueden asignar etiquetas y sobre las que pueden hacer comentarios quienes las consultan, que además pueden descargarlas e incluso incrustarlas en sus blogs o páginas web.

http://www.slideshare.net

11. Este enlace es una Slurl, es decir, que lleva vía Google Maps a una localización dentro de $\mathrm{Se}$ cond Life. Para que funcione es necesario tener una cuenta en Second Life (un avatar) y también que en nuestro ordenador esté instalado el cliente-visor de Second Life (se puede descargar en la web oficial).

http://www.secondlife.com

12. Philip Rosedale in Paris: transcript (Apr. 25, 2008) en SLCamp. Consultado en: 06-06-08. http://slcamp.wordpress.com/2008/05/05/philiprosedale-in-paris-transcript-apr-25-2008/

Francisco López-Hernández, Biblioteca de la Universidad Carlos III, Campus de Colmenarejo, Edificio Menéndez-Pidal, Avenida de la Universidad Carlos III, 22, 28270 Colmenarejo (Madrid). pacol@db.uc3m.es 